\title{
Evaluación de los parámetros de seguridad eléctrica en equipos biomédicos y de instrumentación bajo condiciones ambientales no controladas de laboratorio
}

\author{
Robinson Castillo(1)* y Jaime A. Delgado(1) \\ (1) Centro de Electricidad Electrónica y Telecomunicaciones, Servicio Nacional de Aprendizaje SENA, Grupo de \\ Investigación del CEET - SENA (GICS), Av. Carrera 30 \# 17B-25-Sur, Bogotá D.C., Colombia. \\ (e-mail: rcastillo48@misena.edu.co jadelgado993@misena.edu.co). \\ * Autor a quien debe ser dirigida la correspondencia
}

Recibido May. 3, 2019; Aceptado Jul. 3, 2019; Versión final Sep. 1, 2019, Publicado Feb. 2020

\begin{abstract}
Resumen
Este articulo presenta la evaluación de los parámetros de Seguridad Eléctrica en sus requerimientos generales, para equipos biomédicos y de instrumentación electrónica, de acuerdo a la normatividad de la Comisión Electrotécnica Internacional, IEC, para condiciones ambientales de laboratorio no controladas, como son la temperatura y el porcentaje de humedad relativa. Lo anterior está enfocado a la realización de un protocolo de pruebas de pre-conformidad y detección in situ de fallas en equipos de electro-tecnología. Se evaluaron dieciocho equipos, incluyendo biomédicos y de instrumentación. Luego se realizó el análisis de correlación de las variables ambientales con los parámetros medidos, para determinar la influencia de las variables ambientales en los parámetros de Seguridad Eléctrica. Los resultados muestran que dichos parámetros no presentan correlación con los valores de las variables ambientales medidas.
\end{abstract}

Palabras clave: pruebas de seguridad eléctrica; parámetros eléctricos; variables ambientales; electrotecnología; protocolo de pruebas; detección de fallas

\section{Evaluation of electrical safety parameters in biomedical and instrumentation equipment under uncontrolled environmental laboratory conditions}

\begin{abstract}
This article presents the evaluation of the parameters of Electrical Safety in its general requirements, for biomedical equipment and electronic instrumentation, according to the regulations of the International Electrotechnical Commission, IEC, for uncontrolled environmental laboratory conditions, such as the temperature and the percentage of relative humidity. This evaluation is done to implement a protocol for preconformity tests and in situ fault detection for electro-technology equipment. Eighteen equipment were evaluated, including biomedical and instrumentation. After that a correlation analysis of the environmental variables was performed with the measured parameters, to determine the influence of the environmental variables on the Electrical Safety parameters. The results show that such parameters do not present any correlation with the measured environmental variables.
\end{abstract}

Keywords: electrical safety tests; electrical parameters; environmental variables; electro-technology; test protocol; fault detection 


\section{INTRODUCCIÓN}

El desarrollo de productos eléctricos, electrónicos, tecnológicos y afines "electro-tecnología", exigen del cumplimiento de la normatividad a nivel de Seguridad Eléctrica (SE), estas normas verifican el óptimo funcionamiento del equipo, evitando posibles problemas de Compatibilidad Electromagnética (CEM) entre otros equipos de electro-tecnología (Martyr y Plint, 2007), y principalmente aseguren la integridad a los usuarios finales (Villa et al., 2009; AENOR, 2007). Además de facilitar el comercio y la transferencia tecnológica entre organización y países, al ofrecer una estandarización en la concepción y diseño de estos productos. A nivel internacional existen diferentes entes encargadas de realizar los procesos de regulación y acreditación de los equipos de electro-tecnología en Seguridad Eléctrica, como principal organización se tiene a la Comisión Electrotécnica Internacional (IEC por sus siglas en ingles), la cual establece las normas y regulaciones que debe cumplir cualquier equipo de electro-tecnología.

Para el caso de los países latinoamericanos, los entes de control interno de cada país, asimilan la normatividad internacional y la adaptan para aplicarla en concordancia al mercado y a los acuerdos comerciales firmados, esto permite que equipos importados los cuales no cumplan con la normatividad, no entren a las economías locales, y exige además que los equipos desarrollados en el país de origen, cumplan con la norma, si su intención es ser comercializados a nivel local o internacional. Sin embargo, el reto para los desarrolladores de productos de electro-tecnología locales, es lograr obtener la certificación internacional que les permita entrar a competir en otros mercados, es aquí donde se presentan dos inconvenientes, el primero es el costo que representa el proceso de certificación, el cual en algunos casos puede representar un costo superior al desarrollo del equipo, el segundo inconveniente es la no existencia de laboratorios locales con la certificación internacional de la norma a evaluar, en parte al elevado costo que representa la puesta en marcha y funcionamiento de este tipo de laboratorios (Armstrong y Clough, 2014).

Por lo expuesto anteriormente, se propone la evaluación de los parámetros de SE en equipos biomédicos y de instrumentación electrónica, enunciados en las normas IEC 61010-1 (IEC, 2010) e IEC 60601-1 (IEC, 2007), para la legislación colombiana NTC-60601-1-2 (ICONTEC, 2013), como el realizado por (Pérez et al., 2019), bajo condiciones ambientales de temperatura y de porcentaje de humedad relativa no controladas, pero realizando una medición de las mismas cuando se evalúen los parámetros de Seguridad Eléctrica, como en (Wolffenbuttel y Wijngaards, 2003, Chavez et al., 2005). El objetivo es determinar la influencia de las variables ambientales (Ang et al, 2003; Wang et al., 2007; Brunetto et al., 2010) en los parámetros de Seguridad Eléctrica registrados, con el propósito del desarrollo de un protocolo de medición in situ de equipos electro-tecnología. Este protocolo estaría enfocado en la realización de pruebas de pre-conformidad y de detección de fallas, para equipos que estén en busca de la certificación internacional.

De acuerdo al tipo de equipo de electro-tecnología a evaluar, la IEC especifica una serie de pruebas para verificar su óptimo funcionamiento, como en (Pérez, 2019), para los equipos de instrumentación se evaluó la norma Comisión Electrotécnica Internacional 61010-1 de acuerdo a (IEC, 2010; QuadTech, 2002; Lohbecek, 2004), y para los equipos biomédicos la norma IEC 60601-1 de acuerdo a (IEC, 2007; Salazar y Cuervo, 2013). Los parámetros que evalúa cada una de estas normas en su numeral 1 , son pruebas que garantizan la seguridad e integridad de los usuarios ante choques o descargas eléctricas y deben ser de estricto cumplimiento en el diseño de equipos de electro-tecnología (Martens y So, 2006), el primer parámetro se conoce como: Contacto a Tierra (GB, por sus siglas en inglés) y comprueba la existencia de una conexión a tierra eléctrica. Esto incluye a los metales conductivos expuestos en la superficie del equipo, los cuales deben estar aterrizados (Rappaport, 2012).

El segundo parámetro es el de Resistencia de Aislamiento (IR, por sus siglas en inglés), este parámetro mide la calidad del aislamiento del equipo. Por lo general es el encerramiento, caja o carcasa del equipo, el cual está hecho de material metálico en la mayoría de los casos, el cual ofrece la protección de los componentes del equipo, así como también blindaje ante campos electromagnéticos emitidos o radiados (Fowler, 2000). El tercer parámetro es el de Corrientes de Fuga (LC, por sus siglas en inglés), el cual verifica que la corriente que fluye entre la fuente de corriente alterna y la tierra, no exceda el umbral especificado por la norma (IEC, 2007), de acuerdo al tipo de equipo a evaluar.

Esta prueba es de especial interés en equipos biomédicos (Jiju y Khambatte, 2013; Sezdi, 2009), para este parámetro existen tres tipos de pruebas, en este caso se evalúa la corriente de fuga a tierra, que es la corriente que circula desde la de red de alimentación a lo largo o a través del aislamiento al conductor de protección a tierra como se describe en (IEC, 2010; IEC, 2007; ISO, 2012; Rodríguez, et al, 2013). En la Tabla 1 se muestran los valores umbrales para los tres parámetros de SE que se especifican cada una de las normas en una condición normal (los valores se incrementan en caso de falla). 
Tabla 1: Normas y valores umbrales de parámetros de SE.

\begin{tabular}{|c|l|c|c|c|}
\hline \multirow{2}{*}{ Norma } & \multicolumn{1}{|c|}{ Descripción } & GB & IR & LC \\
\cline { 3 - 5 } & \multicolumn{1}{|c|}{$\begin{array}{c}\text { Resistencia } \\
\text { (en } \mathrm{m} \Omega \text { ) }\end{array}$} & $\begin{array}{c}\text { Resistencia } \\
\text { (en } \mathrm{M} \Omega \text { ) }\end{array}$ & $\begin{array}{c}\text { Corriente } \\
\text { (en } \mathrm{mA} \text { ) }\end{array}$ \\
\hline IEC 61010-1 & $\begin{array}{l}\text { Parte 1: Requerimientos generales de seguridad para } \\
\text { equipos eléctricos de medición, control, uso de laboratorio } \\
\text { e instrumentación. }\end{array}$ & $<100$ & $>100$ & $<0.7$ \\
\hline IEC 60601-1 & $\begin{array}{l}\text { Parte 1: Requerimientos generales de seguridad y } \\
\text { desempeño para equipos eléctricos médicos (biomédicos) }\end{array}$ & $<200$ & $>10$ & $<0.5$ \\
\hline
\end{tabular}

\section{MATERIALES Y MÉTODOS}

Para este estudio se utilizaron 18 equipos pertenecientes al Centro de Eléctrica y Electrónica (CEET) del Servicio Nacional de Aprendizaje (SENA). Los equipos son usados para la formación técnica y tecnológica en biomédica, eléctrica y electrónica.

En la Tabla 2 se da una relación de los equipos biomédicos y de instrumentación usados para las pruebas y se proporciona su identificación durante el desarrollo de las pruebas. Todos los equipos de instrumentación tienen tipo de protección y asilamiento clase 1, lo que comprende, que son equipos alimentados por una fuente externa de alimentación y presentan conexión a tierra por medio de una clavija de conexión de tres pines. La mayoría de equipos biomédicos presentan clasificación de riesgo del tipo BF y CF, la primera se relaciona a equipos que tienen un aditamento con entradas 0 accesorios aplicados al paciente mediante circuitos flotantes que aíslan eléctricamente al paciente, pero no al órgano o sistema que se esté monitoreando. La segunda se relaciona a equipos que tienen un accesorio con un alto grado de protección ante corrientes de fuga, el accesorio se encuentra flotante, y se pueden conectar al órgano o sistema que se esté monitoreando del paciente.

Tabla 2: Relación y características de los equipos de instrumentación y biomédica utilizados para las pruebas.

\begin{tabular}{|l|c|l|c|}
\hline \multicolumn{2}{|c|}{ Instrumentación Electrónica } & \multicolumn{2}{c|}{ Biomédica } \\
\hline Equipo & Identificación & Equipo & Identificación \\
\hline Osciloscopio Análogo & E1 & Máquina de Anestesia & B1 \\
\hline Osciloscopio Digital & E2 & Microscopio Quirúrgico & B2 \\
\hline Fuente de poder Dual & E3 & Desfibrilador Monofásico & B3 \\
\hline Analizador de espectro & E4 & Microcentrífuga & B4 \\
\hline Tablero micro automatización & E5 & Desfibrilador Bifásico & B5 \\
\hline Tablero PLC 2 & E6 & Monitor de Signos Vitales & B6 \\
\hline Empalmadora fibra óptica & E7 & Perfusor & B7 \\
\hline Generador de funciones & E8 & Electrobisturí & B8 \\
\hline Medidor de campo & E9 & Neumoinsuflador & B9 \\
\hline
\end{tabular}

\section{Equipos usados para la medición}

Se utilizó el analizador Chroma® 19032 (CHROMA ATE INC, 2015), el equipo viene pre-configurado con los valores umbrales de medición de cada parámetro, de acuerdo al tipo de norma a evaluar, para este caso las normas IEC 61010-1 y 60601-1 en sus requerimientos generales. La medición de las condiciones ambientales se realizó con el equipo portátil Erasmus ${ }^{\circledR}$ EFA4. Los rangos de medida de temperatura para este equipo son de $-20^{\circ} \mathrm{C}$ a $750^{\circ} \mathrm{C}$, con una incertidumbre en la medida de $\pm 3 \%+2^{\circ} \mathrm{C}$, para el porcentaje de humedad relativa presente en el ambiente (\%HR), sus rangos están entre el $25 \%$ al $95 \%$ con una incertidumbre en la medida de $\pm 5 \%$ HR. Los equipos cuentan con certificados de calibración de fábrica.

\section{Protocolo de Medición}

Para la realización del protocolo de mediciones, se adaptaron los requerimientos que solicitan las normas IEC 61010-1 y 60601-1, como en (Correa, et al., 2017; Badnjević, et al., 2015; Buzduga et al., 2007). El protocolo utilizado fue el siguiente: Se realizó la medición de los parámetros de SE y de condiciones ambientales para cada uno de los equipos, en su lugar de ubicación (in situ). Los equipos de biomédica e instrumentación se encuentran en lugares diferentes. A cada uno de los equipos les fueron realizadas nueve mediciones, las cuales fueron divididas en tres días diferentes, en tres diferentes horas del día, y con espaciamiento de cuatro horas entre estas (8:00, 12:00 y 16:00 horas). En las Figuras 1 y 2 , se muestran el protocolo aplicado a cada uno de los equipos, y la disposición de los equipos de medida para la prueba. 


\section{Tratamiento de los datos de medición}

Se usaron diagramas de cajas (Boxplots), para el tratamiento de los datos obtenidos de las pruebas, estos describen características de los datos, tales como: simetría (tendencia de los datos), dispersión (rango intercuartílico) y datos atípicos (Snecdecor y Cochran, 2014). Se estimó la correlación Pearson (Runger y Montgomery, 2013) de cada uno de los parámetros de SE con los datos ambientales, por medio de la ecuación (1).

1. Ubicar Analizador y Medidor de condiciones ambientales en el lugar donde se encuentra el EBP

2. Realizar la configuración de la norma a evaluar (61010-1 o 60601-1) del Analizador, y las conexiones con el EBP

3. Realizar las pruebas de SE al EBP y registrar la temperatura y el porcentaje de humedad relativa con el EFA4

4. Realizar el registro de los parámetros de SE (GB, IR y LC) en la bitácora de mediciones

5. Apagar el EBP y realizar la desconexión con el Analizador

Fig. 1: Protocolo de pruebas aplicado

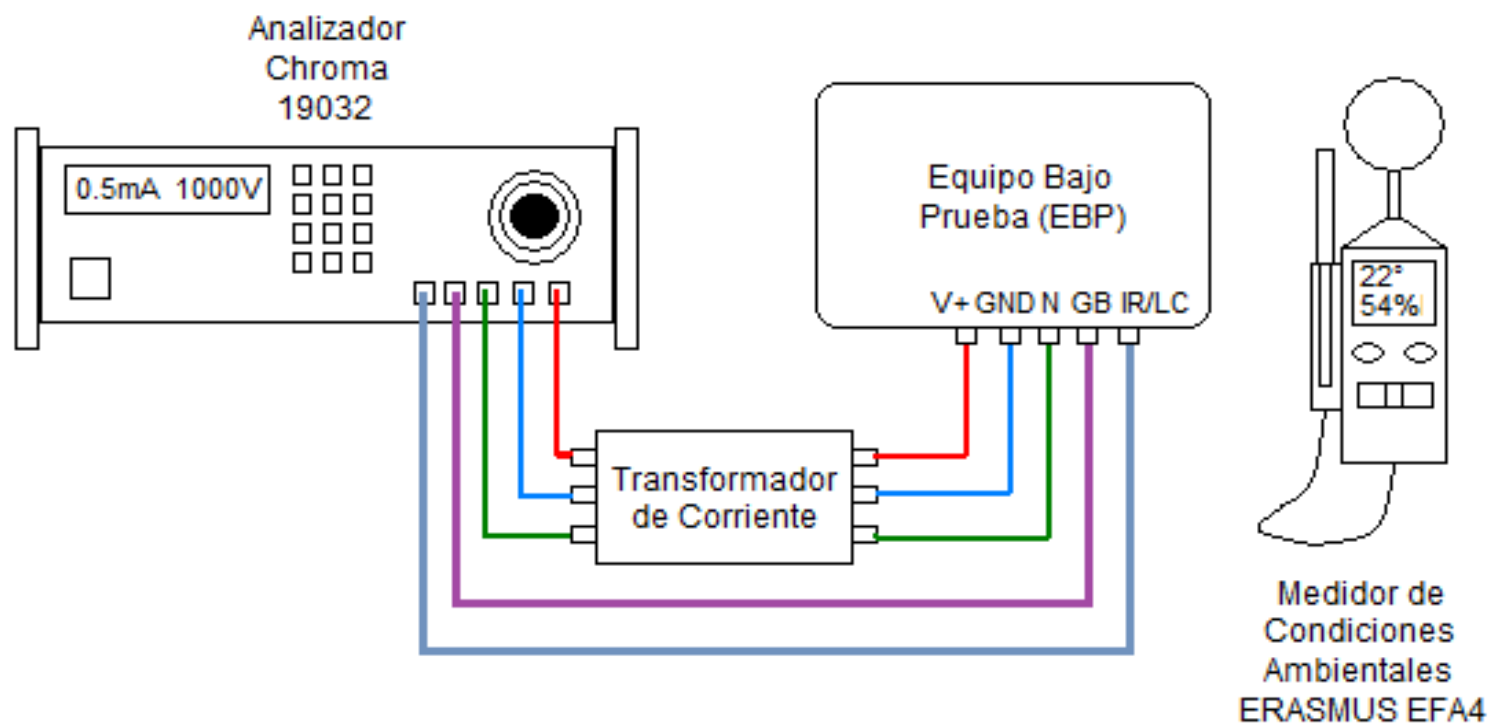

Fig. 2: Esquema general de la disposición de equipos usados durante las pruebas.

$$
\rho(\mathrm{A}, \mathrm{B})=\frac{1}{\mathrm{~N}-1} \sum_{i=1}^{N}\left(\frac{A_{i}-\mu_{A}}{\sigma_{\mathrm{A}}}\right)\left(\frac{B_{i}-\mu_{B}}{\sigma_{\mathrm{B}}}\right)
$$

En esta ecuación, A y B son las variables para el proceso de correlación, tomando A como los datos de medición de cada uno de los parámetros de SE, y B como los datos de medición de cada una de las variables ambientales, $\mu_{\mathrm{A}}$ у $\mu_{\mathrm{B}}$ son las medias calculadas para cada una de las variables $\mathrm{A}$ у $\mathrm{B}$, у $\sigma_{\mathrm{A}}$ у $\sigma_{\mathrm{B}}$ son las desviaciones estándar para A y B respectivamente (Raynal, 2005). 


\section{RESULTADOS}

Para una mejor explicación y discusión, los resultados se presentan en dos secciones: una presenta los resultados de las pruebas de SE para los equipos de instrumentación electrónica, y una segunda sección muestra los resultados de las pruebas de SE para los equipos biomédicos.

\section{Equipos de instrumentación electrónica}

A continuación, se muestran los resultados de las pruebas de SE para los equipos de instrumentación electrónica, se inicia con el parámetro de contacto a tierra (GB), se continua con el parámetro de resistencia de aislamiento (IR) y finalmente con el parámetro de corrientes de fuga (LC), como se mencionó en el parágrafo anterior, para este último parámetro se tomó la medida de corrientes de fuga a tierra. Se muestra también la medida de temperatura y del porcentaje de humedad relativa con cada uno de los parámetros.

\section{Contacto a tierra $(G B)$ y variables ambientales}

En la Figura 3 se muestra el diagrama de cajas del parámetro GB y las variables ambientales. En el diagrama se puede observar que solo dos de los equipos superaron el valor límite de $100 \mathrm{~m} \Omega$ establecido por la norma IEC 61010-1, para una condición normal (CN). Los equipos fueron: E7 (Empalmadora fibra óptica) y E8 (Generador de funciones). A diferencia del resto de los equipos, para los cuales gran parte de las mediciones se concentran en su mediana (línea en negro), los equipos E7 y E8 presentan una medida de dispersión en sus datos, lo cual se muestra en el cálculo de sus rangos intercuartílicos, donde para el equipo E7 se obtuvo un valor de $R_{Q 3-Q 1}=16.8$, y $R_{Q 3-Q 1}=29.82$ respectivamente, además el equipo $E 7$ presentó un valor atípico de $152 \mathrm{~m} \Omega$ en una de sus mediciones.
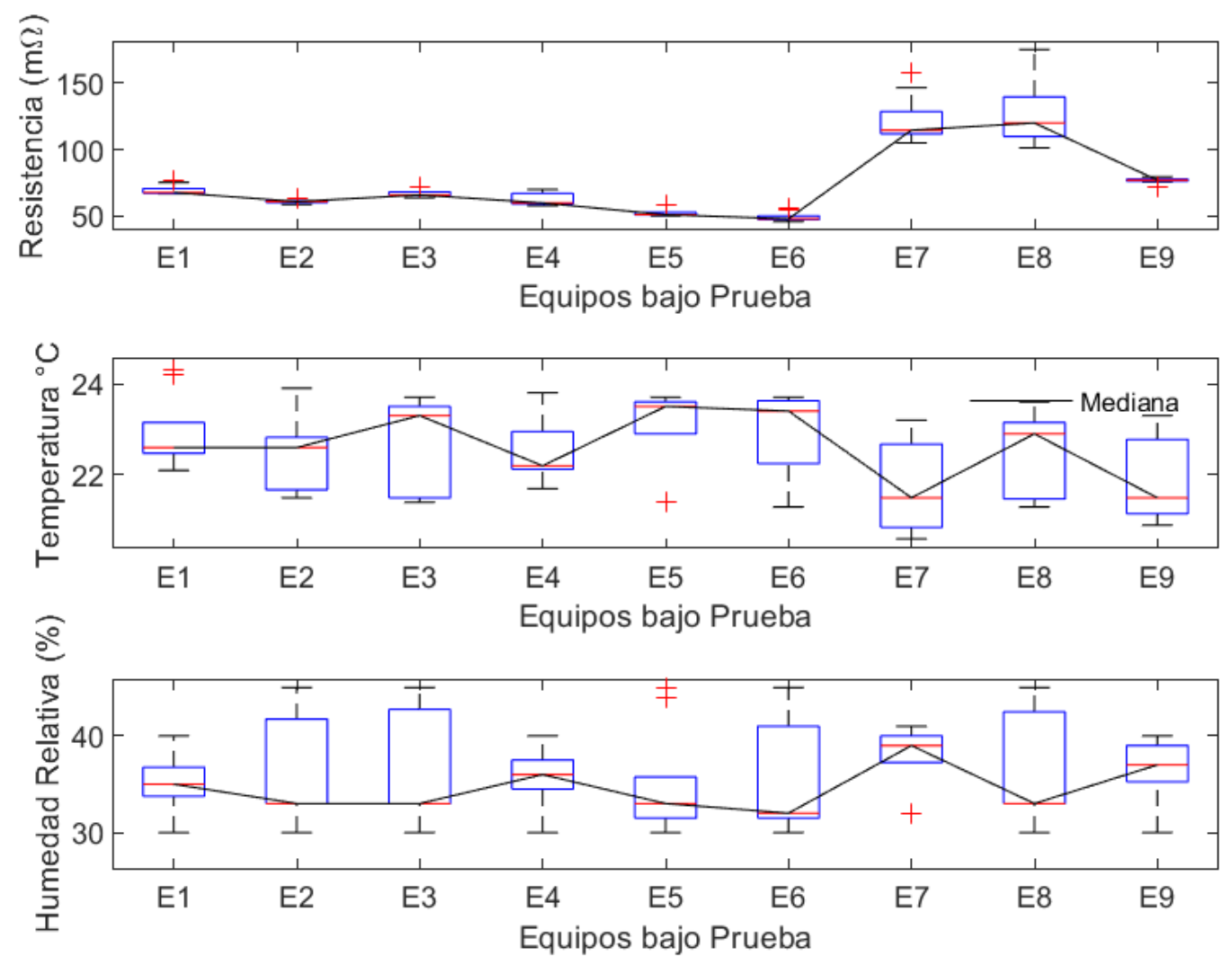

Fig. 3: Parámetro GB y variables ambientales medidas para los equipos de instrumentación.

En la Tabla 3 se muestran los valores de correlación obtenidos del parámetro GB con las variables ambientales medidas a partir de (1). El equipo E5 (Tablero micro automatización), presentó una correlación positiva cercana a 1 para las dos variables ambientales medidas, y el equipo E9 (Medidor de campo) presentó correlación positiva cercana a 1, para la variable de porcentaje de humedad relativa. El resto de equipos no presentaron valores representativos. 
Tabla 3: Parámetro GB y correlación con variables ambientales medidas.

\begin{tabular}{|c|c|c|c|c|c|c|c|c|c|}
\hline $\begin{array}{c}\text { Parámetros } \\
\text { Correlacionados }\end{array}$ & E1 & E2 & E3 & E4 & E5 & E6 & E7 & E8 & E9 \\
\hline GB y Temperatura & 0.256 & -0.057 & 0.694 & 0.510 & 0.980 & 0.437 & 0.347 & 0.320 & 0.340 \\
\hline GB y \% H. Relativa & 0.693 & 0.036 & 0.567 & 0.005 & 0.988 & 0.648 & 0.195 & 0.273 & 0.832 \\
\hline
\end{tabular}

\section{Resistencia de aislamiento (IR) y variables ambientales}

En la Figura 4 se muestra los resultados para el parámetro IR y las variables ambientales medidas. Todos los equipos superaron el valor umbral de $100 \mathrm{M} \Omega$ que especifica la norma IEC 61010-1. Sin embargo, los equipos E2 (Osciloscopio Digital), E7 (Empalmadora fibra óptica) y E8 (Generador de funciones) presentaron dispersión en las mediciones del parámetro, con relación a su mediana, en el cálculo de sus rangos intercuartílicos, se obtuvo para los equipos E2, E7 y E8 los siguientes valores: $R_{Q 3-Q 1}=3.2, R_{Q 3-Q 1}=3.58$, $R_{Q 3}$ Q1 $=3$.2. Se presentaron además valores atípicos para los equipos E5 (Tablero micro automatización) y E6 (Tablero PLC 2).
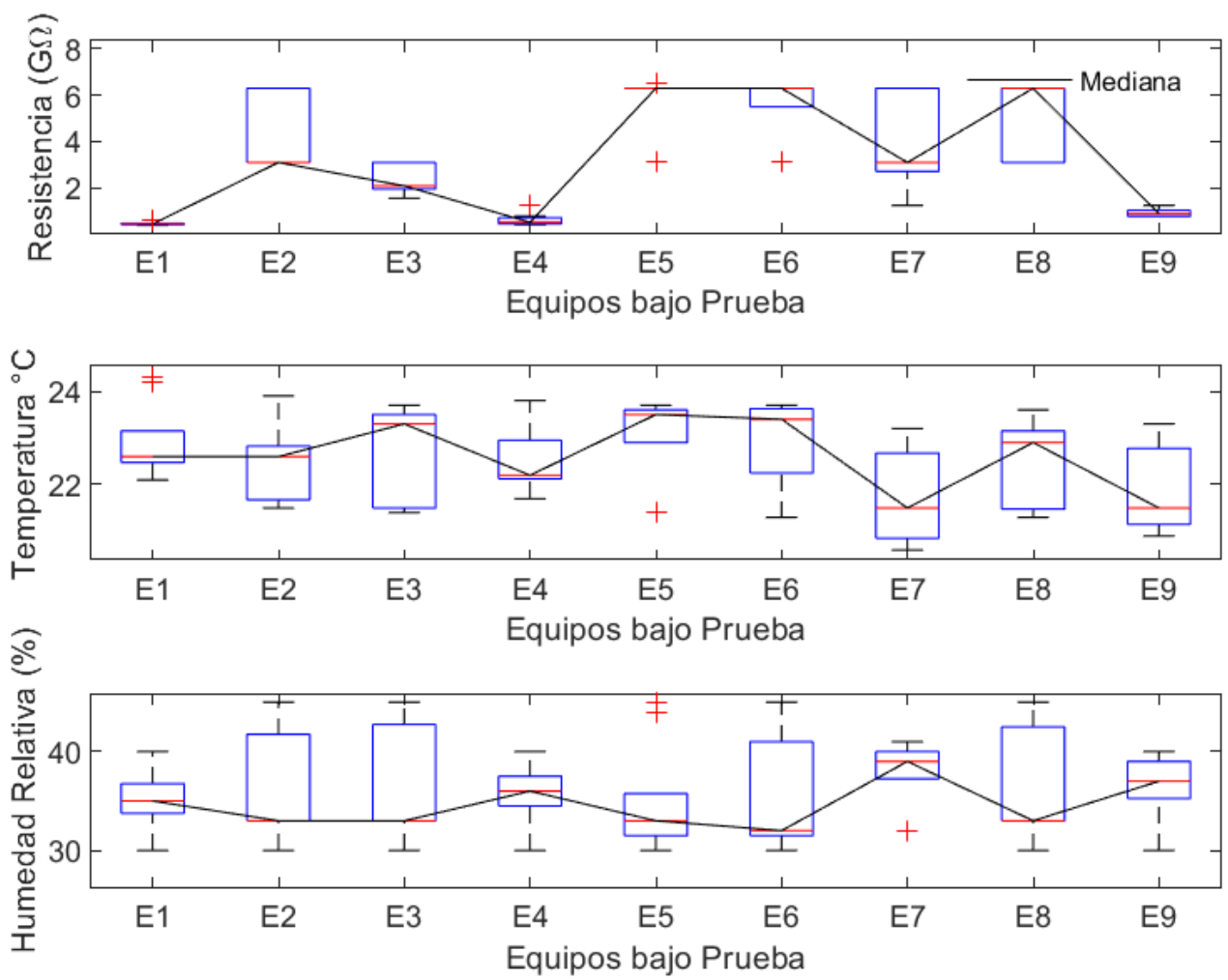

Fig. 4: Parámetro IR y variables ambientales medidas para los nueve equipos de instrumentación.

En la Tabla 4. se puede observar, que para el parámetro IR, solo el equipo E3 (Fuente de poder Dual) presentó un valor de correlación positivo cercano a 1 , con respecto a la variable de temperatura, el resto de equipos no presentaron ningún tipo de correlación entre el parámetro IR y las variables ambientales medidas, por lo tanto, se puede determinar que no se presenta correlación entre las variables ambientales medidas y el parámetro IR, para los equipos de instrumentación.

Tabla 4: Parámetro IR y correlación con variables ambientales medidas.

\begin{tabular}{|c|c|c|c|c|c|c|c|c|c|}
\hline $\begin{array}{c}\text { Parámetros } \\
\text { Correlacionados }\end{array}$ & E1 & E2 & E3 & E4 & E5 & E6 & E7 & E8 & E9 \\
\hline IR y Temperatura & 0.675 & 0.466 & 0.743 & 0.007 & 0.151 & 0.181 & 0.180 & 0.420 & 0.311 \\
\hline IR y \% H. Relativa & 0.525 & 0.616 & 0.617 & 0.475 & 0.293 & 0.188 & 0.147 & 0.312 & 0.561 \\
\hline
\end{tabular}

Corrientes de fuga (LC) y variables ambientales

En la Tabla 5 se muestran los resultados de correlación aplicando (1), donde sólo el equipo E8 (Generador de funciones) presentó un valor cercano a 1, para el parámetro LC y las variables ambientales medidas, sin 
embargo, el equipo E8 cumple con el valor del parámetro evaluado por la norma. Para el resto de equipos no se presentaron valores de correlación representativos.

Tabla 5: Parámetro LC y correlación con variables ambientales medidas.

\begin{tabular}{|c|c|c|c|c|c|c|c|c|c|}
\hline $\begin{array}{c}\text { Parámetros } \\
\text { Correlacionados }\end{array}$ & E1 & E2 & E3 & E4 & E5 & E6 & E7 & E8 & E9 \\
\hline LC y Temperatura & 0.015 & 0.141 & 0.018 & 0.423 & 0.046 & 0.337 & 0.652 & 0.846 & 0.232 \\
\hline LC y \% H. Relativa & 0.400 & 0.255 & 0.050 & 0.154 & 0.280 & 0.570 & 0.245 & 0.943 & 0.022 \\
\hline
\end{tabular}

En la Figura 5 se muestra el diagrama de cajas del parámetro LC y las variables ambientales. En el diagrama se puede observar que todos los equipos presentaron valores inferiores al límite de $0.7 \mathrm{~mA}$, que especifica la norma IEC 61010-1, para la prueba de corrientes de fuga a tierra, en CN. Al realizar el cálculo del rango intercuartílico, ninguno de los equipos presentó una dispersión representativa en sus datos de medición. Se presentó un valor atípico en la medición para el equipo E4 (Analizador de espectro) de $0.82 \mathrm{~mA}$.
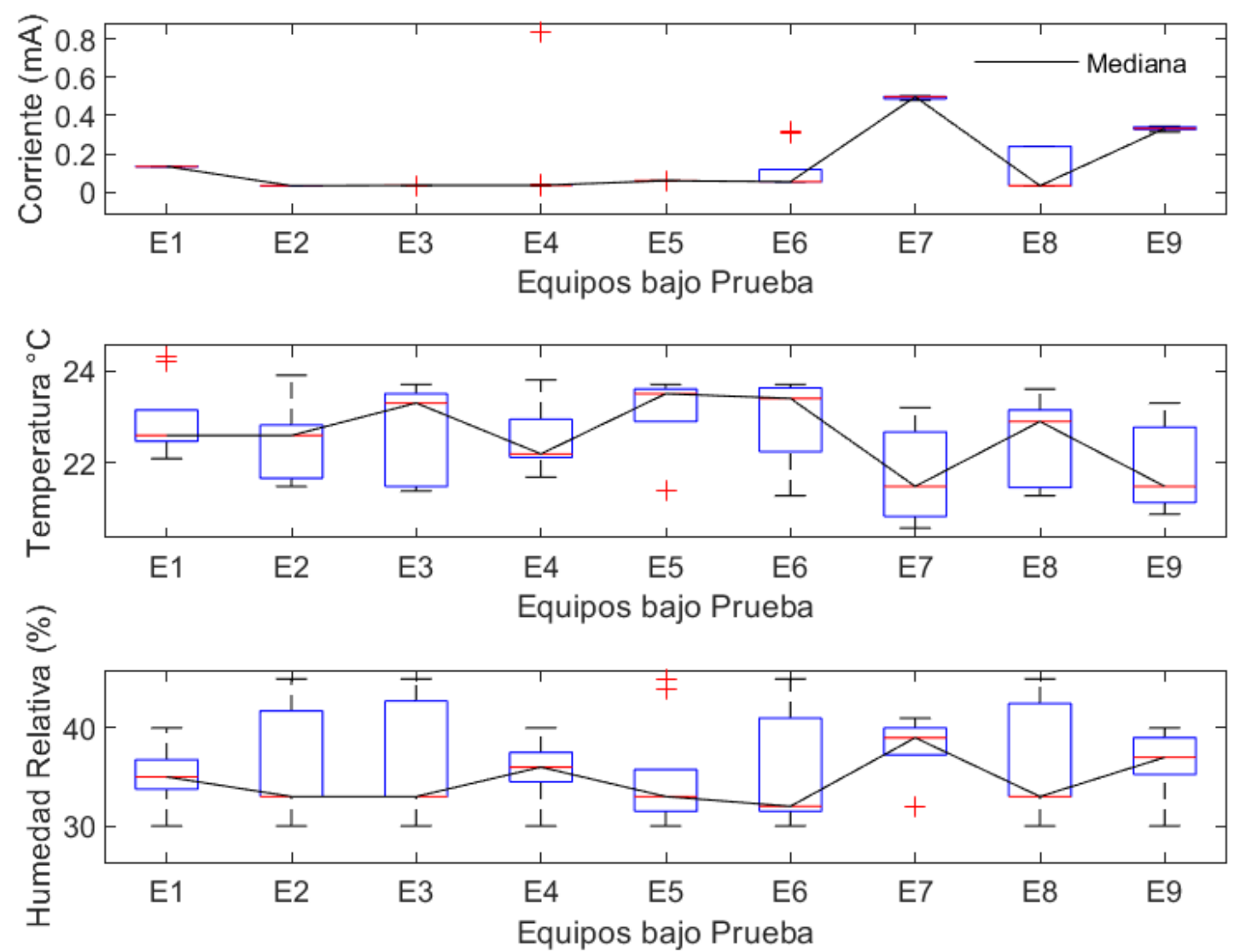

Fig. 5: Parámetro LC y variables ambientales medidas para los nueve equipos de instrumentación.

\section{Equipos biomédicos}

A continuación se muestran los resultados de las pruebas de SE para los equipos biomédicos, al igual que para los equipos de instrumentación electrónica, se inicia con el parámetro de contacto a tierra (GB), que a diferencia de los equipos de instrumentación electrónica, el parámetro no debe sobrepasar el valor límite de $200 \mathrm{~m} \Omega$, se continua con el parámetro de resistencia de aislamiento (IR), que para los equipos biomédicos, el valor del parámetro debe ser superior a $10 \mathrm{M} \Omega$, y finalmente con el parámetro de corrientes de fuga (LC), donde el valor límite para los equipos biomédicos no debe sobrepasar el valor de $0.5 \mathrm{~mA}$, al igual que para los equipos de instrumentación electrónica, se tomó la medida de corrientes de fuga a tierra, también se muestran las medidas de temperatura y del porcentaje de humedad relativa.

\section{Contacto a tierra $(G B)$ y variables ambientales}

En la Tabla 6 se muestra que el parámetro GB no presentó correlación con las variables ambientales medidas al aplicar (1). En la Figura 6 se muestran los resultados para el parámetro GB y las variables ambientales 
medidas. Todos los equipos cumplieron con el valor límite de $200 \mathrm{~m} \Omega$ establecido por la norma IEC 60601-1, y sus mediciones del parámetro se concentran en su mediana, se presentaron valores atípicos de $149.6 \mathrm{~m} \Omega$ y $123.2 \mathrm{~m} \Omega$ para los equipos B2 (Microscopio Quirúrgico) y B6 (Monitor de Signos Vitales).

Tabla 6: Parámetro GB y correlación con variables ambientales medidas.

\begin{tabular}{|c|c|c|c|c|c|c|c|c|c|}
\hline $\begin{array}{c}\text { Parámetros } \\
\text { Correlacionados }\end{array}$ & B1 & B2 & B3 & B4 & B5 & B6 & B7 & B8 & B9 \\
\hline GB y Temperatura & 0.272 & 0.625 & 0.276 & 0.250 & 0.217 & 0.393 & 0.248 & 0.430 & 0.122 \\
\hline GB y \% H. Relativa & 0.301 & 0.620 & 0.327 & 0.292 & 0.243 & 0.528 & 0.230 & 0.504 & 0.145 \\
\hline
\end{tabular}
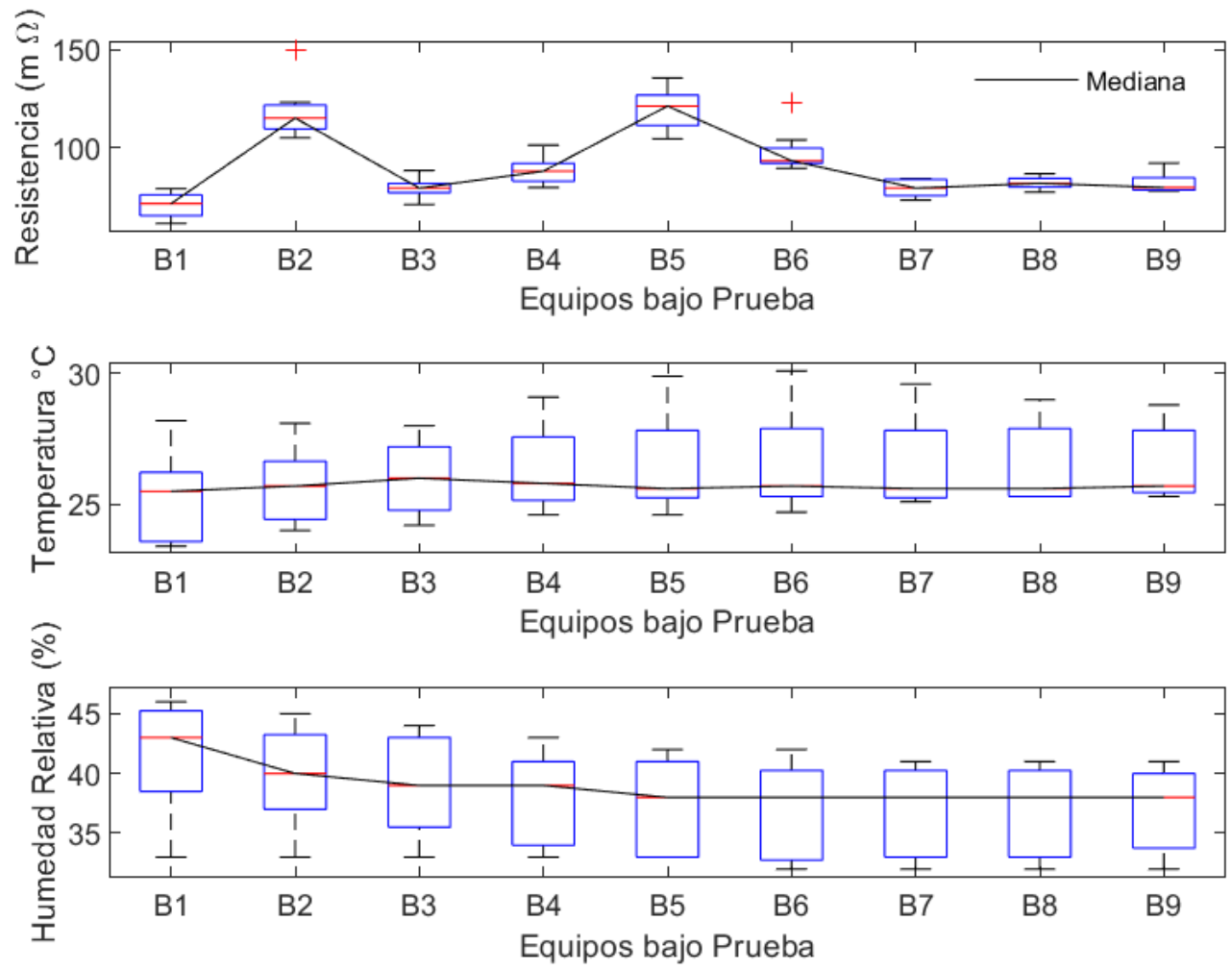

Fig. 6: Parámetro GB y variables ambientales para los nueve equipos biomédicos.

\section{Resistencia de aislamiento (IR) y variables ambientales}

En la Tabla 7. se muestran los resultados de la correlación del parámetro IR y las variables ambientales, los equipos B3 (Desfibrilador Monofásico), B4 (Microcentrífuga) y B6 (Monitor de Signos Vitales) presentaron valores positivos cercanos a 1, para las dos variables medidas, los equipos B7 (Perfusor) y B9 (Electrobisturí) sólo para la variable de porcentaje de humedad relativa. Aunque cinco de los nueve equipos evaluados presentan una correlación con el parámetro IR con al menos una de las variables ambientales, o ambas, esta correlación no afecto el valor del parámetro IR solicitado por la norma.

Tabla 7: Parámetro IR y correlación con variables ambientales medidas.

\begin{tabular}{|c|c|c|c|c|c|c|c|c|c|}
\hline $\begin{array}{c}\text { Parámetros } \\
\text { Correlacionados }\end{array}$ & B1 & B2 & B3 & B4 & B5 & B6 & B7 & B8 & B9 \\
\hline IR y Temperatura & 0.452 & 0.570 & 0.944 & 0.806 & 0.411 & 0.717 & 0.580 & 0.507 & 0.615 \\
\hline IR y \% H. Relativa & 0.337 & 0.529 & 0.961 & 0.788 & 0.452 & 0.846 & 0.784 & 0.569 & 0.741 \\
\hline
\end{tabular}

En la Figura 7 se muestran los resultados del parámetro IR y las variables ambientales medidas. Todos los equipos superaron el valor umbral de $10 \mathrm{M} \Omega$ que especifica la norma IEC 60601-1 y sus mediciones se concentran en su mediana, a excepción del equipo B2 (Microscopio Quirúrgico) que presentó dispersión en sus mediciones, el cálculo de su rango intercuartílico presentó un valor de RQ3-Q1=3.2 por encima de su mediana. Los equipos B5 (Desfibrilador Bifásico), B8 (Electrobisturí) y B9 (Neumoinsuflador) presentaron valores atípicos en sus mediciones: $6.2 \mathrm{G} \Omega$ para el equipo $\mathrm{B} 5,2.3 \mathrm{G} \Omega$ y $0.56 \mathrm{G} \Omega$ para el equipo $\mathrm{B} 8$, y para el equipo B9 los valores de $2.27 \mathrm{G} \Omega$ y $1.13 \mathrm{G} \Omega$. 


\section{Corrientes de fuga (LC) y variables ambientales}

En la Tabla 8 se muestra que los equipos B3 (Desfibrilador Monofásico), B4 (Microcentrífuga) y B6 (Monitor de Signos Vitales) no presentaron correlación del parámetro LC y las variables ambientales medidas, al aplicar (1), caso contrario al resto de equipos, los cuales presentaron correlación en las dos variables ambientales medidas, sin embargo, todos los equipos cumplieron con el valor del parámetro LC solicitado por la norma.
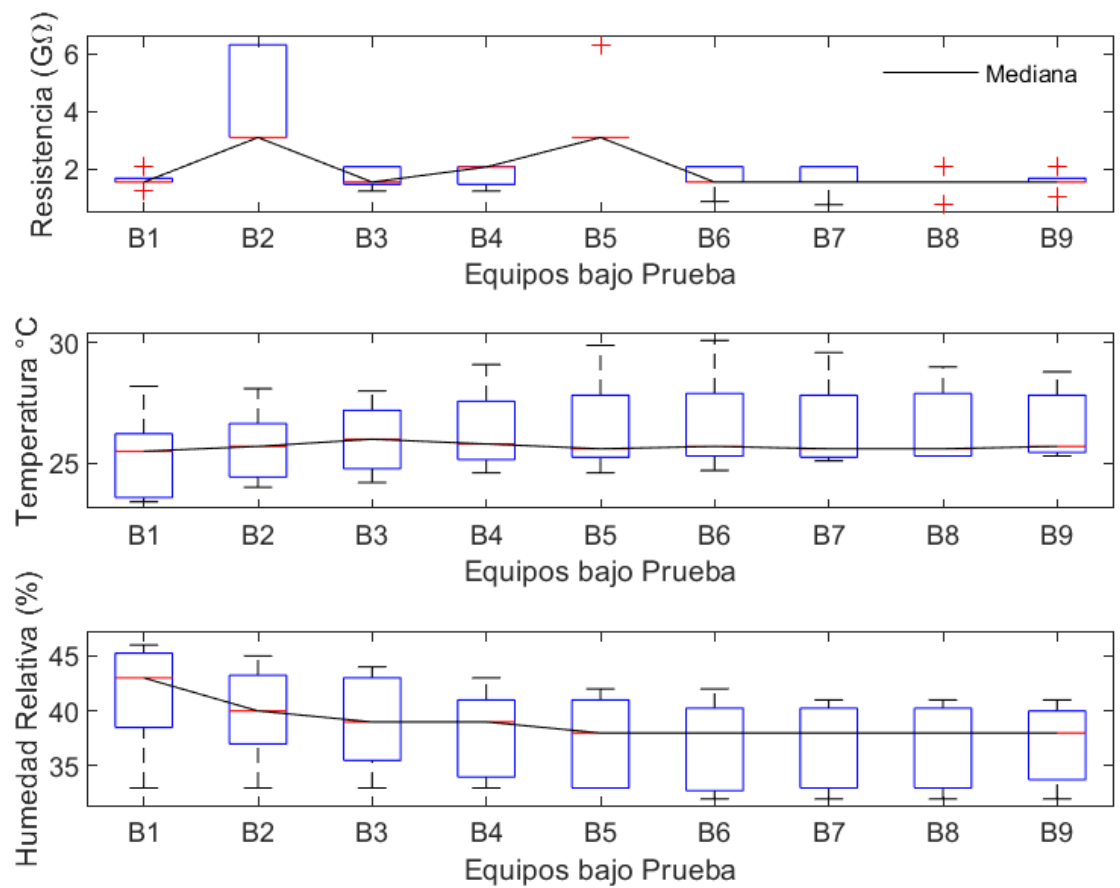

Fig. 7: Parámetro IR y variables ambientales medidas para los nueve equipos biomédicos.

Tabla 8: Parámetro LC y correlación con variables ambientales medidas.

\begin{tabular}{|c|c|c|c|c|c|c|c|c|c|}
\hline $\begin{array}{c}\text { Parámetros } \\
\text { Correlacionados }\end{array}$ & B1 & B2 & B3 & B4 & B5 & B6 & B7 & B8 & B9 \\
\hline LC y Temperatura & 0.747 & 0.737 & 0.531 & 0.298 & 0.769 & 0.024 & 0.696 & 0.833 & 0.736 \\
\hline LC y \% H. Relativa & 0.777 & 0.677 & 0.488 & 0.463 & 0.817 & 0.118 & 0.641 & 0.821 & 0.723 \\
\hline
\end{tabular}
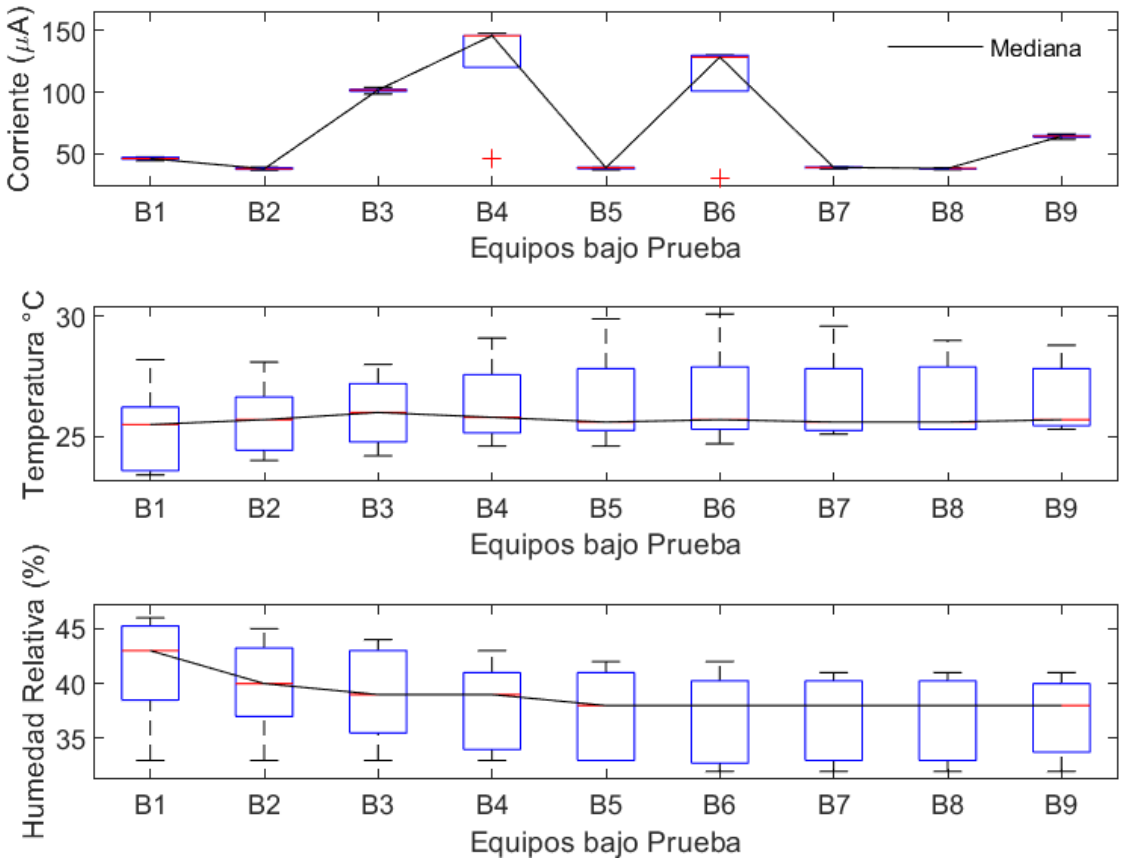

Fig. 8: Parámetro LC y variables ambientales medidas para los nueve equipos biomédicos. 
En la Figura 8 se muestra el diagrama de cajas del parámetro LC y las variables ambientales. En el diagrama se puede observar que todos los equipos presentaron valores inferiores al límite de $0.5 \mathrm{~mA}$, que especifica la norma IEC 60601-1, para la prueba de Corrientes de fuga a tierra en CN. Se presentó dispersión en la medida, para los equipos B4 (Microcentrífuga) y B6 (Monitor de Signos Vitales), donde sus rangos intercuartílicos fueron: para $B 4$ se obtuvo $R_{Q 3-Q 1}=25.82$ y para $B 6$ se obtuvo $R_{Q 3-Q 1}=29.05$. También para estos equipos se observaron valores atípicos de: $50 \mu \mathrm{A}$ y $43.6 \mu \mathrm{A}$.

\section{DISCUSIÓN FINAL}

A lo largo de este artículo se presentó un estudio sobre la evaluación de los parámetros de SE en el lugar de ubicación de los equipos (in situ), por lo general son lugares donde no se presentan condiciones controladas de laboratorio (ambientes de trabajo y practica estudiantil), el objetivo era determinar la influencia de la temperatura y el porcentaje de humedad relativa en los parámetros que las normas IEC 61010-1 y 60601-1, en sus requerimientos generales, evalúa para determinar el comportamiento y la seguridad que ofrece el equipo al usuario. Los parámetros evaluados fueron: contacto a tierra (GB), resistencia de aislamiento (IR) y corrientes de fuga (LC), para el caso de corrientes de fuga, sólo se evaluó el caso de corrientes de fuga a tierra.

Los resultados mostraron que, de los dieciocho equipos evaluados, sólo dos no cumplieron con el valor del parámetro estipulado por la norma (88.8\% de los equipos). Sin embargo, el no cumplimiento del valor del parámetro, no tuvo correlación con las variables ambientales medidas durante la prueba. Con referencia a los equipos biomédicos, siete presentaron correlación de alguno de los parámetros de SE con las variables ambientales, pero el valor de cada uno de los parámetros evaluados, cumplió con los valores solicitados por la norma. De los dieciocho equipos evaluados, los equipos de instrumentación electrónica presentaron correlación con alguna de las variables ambientales, en ningún caso en las dos variables ambientales medidas, y siempre fueron equipos diferentes. Para los equipos biomédicos, los parámetros IR y LC, si presentaron correlación con una o con las dos variables ambientales medidas. Sin embargo, todos los equipos cumplieron con el valor por debajo del umbral que especifica la norma.

Con respecto a las condiciones ambientales medidas, estas no mostraron una tendencia hacia un valor característico, en relación al ambiente donde se realizó dicha medición. Y ninguno de los valores medidos sobrepasaron los límites de trabajo que se especifican en la hoja de datos de cada uno de los equipos. Por lo tanto, es factible la implementación del protocolo propuesto en este estudio, para la medición de parámetros de SE in situ, enfocado en pruebas de precertificación. Equipos como la Máquina de Anestesia (B1), la cual tiene un peso de136kg, el Microscopio Quirúrgico (B2), el cual pesa $298 \mathrm{~kg}$ y el Tablero de micro automatización (E5) con un peso de 66kg, pueden ser evaluados en su lugar de trabajo con este tipo de protocolo, disminuyendo costos hacia las pruebas de precertificación o mantenimiento, ya que no sería necesario el proceso de transporte a un laboratorio de condiciones controladas.

Aunque es necesario el desarrollo de más pruebas, con un mayor número de muestras por equipo, es factible la implementación del protocolo de medición de parámetros de SE, como el desarrollado en este artículo, para el posterior desarrollo de pruebas de precertificación en equipos electro-electrónicos de manufactura nacional, enfocado en el hecho de que, al someterse a las mismas pruebas, en un laboratorio certificado, este pueda obtener su certificación.

\section{CONCLUSIONES}

De acuerdo al trabajo presentado y a los resultados obtenidos, se pueden plantear las siguientes conclusiones principales:

i) La gran mayoría de las mediciones de los parámetros de SE, tanto para los equipos biomédicos, como para los equipos de instrumentación, se concentraron en su mediana de datos, con valores mínimos en sus rangos intercuartílicos.

ii) Los diagramas de cajas o Boxplots, permiten fácilmente determinar de manera gráfica la presencia de tendencias y dispersión de los datos medidos.

iii) Los resultados muestran que los parámetros de SE evaluados, para equipos biomédicos, como para equipos de instrumentación, aplicando las normas IEC 60601-1 e IEC 61010-1, en sus requerimientos generales, no presentan correlación con los valores de las variables ambientales medidas in situ, como la temperatura y el porcentaje de humedad relativa. 
iv) El protocolo implementado, mostró buenos resultados para la evaluación de los requerimientos generales de las normas IEC 60601-1 e IEC 61010-1, en equipos biomédicos y de instrumentación.

v) Este protocolo puede ser adaptado a otras normas IEC u otros estándares internacionales, como el Underwriters Laboratories (UL).

vi) El protocolo implementado permite realizar la medición de parámetros de SE, en equipos que, por su tamaño, peso o ubicación remota, sea dificultoso su transporte a un laboratorio especializado.

\section{AGRADECIMIENTOS}

Este trabajo fue desarrollado por el Centro de Electricidad, Electrónica y Telecomunicaciones (CEET) por medio del proyecto Inmunidad Electromagnética, los autores extienden su agradecimiento al Sistema de investigación, desarrollo tecnológico e innovación del SENA (SENNOVA) quienes financiaron la publicación de este trabajo.

\section{REFERENCIAS}

Martyr, A., Plint, M., Electrical design considerations, Engine Testing (Third Edition) Theory and Practice, 3ra ed, Butterworth-Heinemann, pp 197-215 Elsevier, Amsterdam, Holanda (2007)

Villa, W., Rodríguez, J., Valencia, J., Álvarez, J., Cálculo del Campo Magnético en el Interior de Edificaciones con Sistemas de Protección Contra Rayos, doi: 10.1612/inf.tecnol.4063it.08, Inf. Tecnol. 20(3), $45-54$ (2009).

AENOR, AENOR, Uso y referencia a normas ISO e IEC en la reglamentación técnica, Madrid, España (2007)

Armstrong, K., Clough, C., Why few (if any) medical devices comply with their EMC standard, and what can be done about it, 2014 IEEE International Symposium on Electromagnetic Compatibility (EMC), 1-6, Raleigh, NC, USA 4-8 Aug. (2014)

Pérez, J., Rivera, J., Trujillo, E., Redundancia en Redes de Comunicación para la Automatización y Protección de Sistemas de Potencia Eléctrica con IEC 61850, doi: http://dx.doi.org/10.4067/S0718-07642019000100075, Inf. Tecnol. 30(1), 75-86 (2019).

Badnjević, A., Gurbeta, L., Bošković, D., Džemić, Z., Medical devices in legal metrology, 2015 4th Mediterranean Conference on Embedded Computing (MECO), 1-3, Budva, Montenegro 14-18 June (2015)

CHROMA ATE INC, Why Perform Electrical Safety Testing. Chroma Systems Solutions, acceso Junio 10 2015), China (2015)

Correa, M., Villalba, M. P., García, J. H., Protocolos para evaluación de desempeño en equipos médicos, doi: https://doi.org/10.24050/19099762.n22.2017.1185, Revista Ingeniería Biomédica, 11(22), 65-71 (2017)

Fowler, K., Grounding and shielding. II. Grounding and return, doi: 10.1109/5289.846264, IEEE Instrumentation \& Measurement Magazine, 3(2), 45 - 48 (2000)

IEC 60601-1, International Electrotechnical Commission, Medical electrical equipment - Part 1: General requirements for basic safety and essential performance, 765-777, Ginebra-Suiza (2007)

IEC 61010-1, International Electrotechnical Commission, Safety requirements for electrical equipment for measurement, control, and laboratory use - Part 1: General requirements, Ginebra-Suiza (2010)

Instituto Colombiano de Normas Técnicas (ICONTEC), NTC-IEC 60601-1-2, Equipos Electromédicos. Parte 1: Requisitos generales de seguridad. Sección: 1: Norma Colateral. Requisitos de seguridad para sistemas electromédicos, https://tienda.icontec.org/wp-content/uploads/pdfs/NTC-IEC60601-1-1.pdf, Colombia (2013)

Jiju, K., Sreekumari, B., Khambatte, N., 2011 IEEE International Symposium on Medical Measurements and Applications, 1-5, Bari, Italy 30-31 May (2011)

Sezdi, M., Electrical safety, Bioelectronics and Medical Devices: From Materials to Devices - Fabrication, Applications and Reliability, Woodhead Publishing Series in Electronic and Optical Materials, pp 331-353 Elsevier, Estanbul, Turquia (2019)

Lohbecek, D., Safety isolation protects users and electronic, National Instrument (2004)

Martens, J., Johnson. G. \& So. P, Design considerations for consumer products utilizing high voltage, IEEE Symposium on Product Safety Engineering, 1-6, Irvine, CA, USA 23-24 Oct (2006)

Wolffenbuttel, R., Wijngaards, D., Study on temperature stability improvement of on-chip reference elements using integrated Peltier coolers, doi: 10.1109/TIM.2003.810004, IEEE Transactions on Instrumentation and Measurement, 52(2), 478-482 (2003)

Chavez, J., Garcia, M., Salazar, J., Tome, J., Mitrani, D., Methodology for extracting thermoelectric module parameters, doi: 10.1109/TIM.2005.851473, IEEE Transactions on Instrumentation and Measurement, 54(4), 1548-1552 (2005) 
Ang, C., Chua, S., Liu, L., Behavior and frequency dependence of AC-DC high voltage transfer standard under humidity step changes, doi: 10.1109/TIM.2003. 811566, IEEE Transactions on Instrumentation and Measurement, 52(2), 363-366 (2003)

Wang, Y., Simonson, G., Besant, R., Shang, W., Transient Humidity Measurements: Part II-Determination of the Characteristics of an Interactive Device, doi: 10.1109/TIM.2007.894882, IEEE Transactions on Instrumentation and Measurement, 56(3), 1080-1086 (2007)

Brunetto, P., Fortuna, L., Giannone, P., Graziani, S., Strazzeri, S., Static and Dynamic Characterization of the Temperature and Humidity Influence on IPMC Actuators, doi:10.1109/TIM.2009.2026613, IEEE Transactions on Instrumentation and Measurement, 59(4), 893-908 (2010)

QuadTech, Electrical Safety Testing Reference Guide, 4ta edición, USA 2002

Rappaport, E., Grounding vs. bonding what the National Electrical Code does not explain, 48th IEEE Industrial \& Commercial Power Systems Conference, 1-4, Louisville, KY, USA 20-24 May (2012)

ISO 14971, International Organization for Standarization, Medical Devices - Application of Risk Management to Medical Devices, Corrected version 2007-10-01, Ginebra-Suiza (2012)

Rodriguez, S., Rodriguez, C., Bruni, R., Vanella, O., Development of a leakage current meter using differential method, doi: 10.1109/TLA.2013.6502794, IEEE Latin America Transactions, 11(1), 149 - 154 (2013)

Runger, G., Montgomery, D., PROBABILIDAD Y ESTADISTICA APLICADAS A LA INGENIERIA, 2da ed, 325-338, Limusa Wiley, Mexico (2013)

Raynal, J., Estimadores de Momentos de Probabilidad Pesada para la Distribución General de Valores Extremos para Máximos, doi: http://dx.doi.org/10.4067/S0718-07642005000100011, Inf. Tecnol. 16(1), 75-82 (2005).

Salazar, A. J., Cuervo, D., Protocolo de pruebas de seguridad eléctrica para equipos electromédicos: caso de estudio de equipos de telemedicina, ISSN. 0121-4993, Revista de Ingeniería, 38(1), 27-32 (2013)

Snecdecor, G., Cochran, W., Statistical Methods, 1ra ed, 136-145, Wiley, India, (2014) 